\title{
Cross-National Comparison of Twitter Use between South Korea and Japan: An Exploratory Study
}

\author{
Seong Eun Cho \\ Korea Information Society Development Institute \\ Gwacheon, 427-710, South Korea \\ Han Woo Park (Corresponding author) \\ Department of Media and Communication \\ YeungNam University, Kyungsan, 712-749, South Korea
}

\begin{abstract}
This study compared cross-national Twitter use between Korea and Japan. The main exploratory variables were a) cultural traits and b) disclosure of geographic information. Twitter use was measured by the degree of reciprocity and the numbers of Tweets, followings, and followers. Data were collected using API-based software and analyzed with independent samples t-tests. Content analysis was conducted to validate the findings. The results indicate that Korean and Japanese users employ their own communication strategies reflecting their cultural orientation.
\end{abstract}

Keywords: Cross-National, Japan, Korea, Profile Analysis, Social Media, Twitter

\section{INTRODUCTION}

Recent years have witnessed the rapid development of communication technology. In particular, the worldwide proliferation of global social media (e.g., Twitter) has made it easier for individuals to connect with others and share information beyond physical boundaries and thus promote the globalization of social networks and cultures. Globalization not only facilitates the assimilation of nations and cultures but also fosters salient local cultures in terms of media use and communication practice [7]. Therefore, although people share their daily lifestyles worldwide, they also perceive in part, differences between their local culture and others.

Given a social group, a culture is formed for a long stretch of time and accordingly, culture can be defined at diverse levels, such as individual, organizational, or national [2]. Regarding these diverse cultural levels, recent research has argued that rapid globalization has decreased the significance of national culture and increased the attention to individual-level cultural aspects ([14], [16]). Recent studies have indeed narrowed down the level, not at a national level but at an individual or organizational level: for example, they have explored

\footnotetext{
* Corresponding author,Email: hanpark@ynu.ac.kr Manuscript received Jun. 05, 2012; revised Aug 3, 2012; accepted Aug 13, 2012
}

communication strategies and information seeking behaviors in an age group or an organization ([6], [10], [11]).

However, we have attempted to extend social media research by providing a cross-national comparison of Twitter use. Twitter is one of the most remarkable Internet channels for social communication and information seeking. Scholars have noted the proliferation of Twitter and examined the effect of this communication channel on social relationships and information sharing. Despite a number of studies exploring this topic, few studies have provided cross-national comparisons, focusing only on contexts-occurring communication and information behaviors. For this, we adopted Korean and Japanese samples because the two nations are renowned as well-established IT (Information Technology) countries. Moreover, since the two countries have readily been assessed as having similar national cultures, the cross-national comparison between them will contribute to revisiting and sophisticating the existing cultural dimension, such as individualism-collectivism, which while robust, is criticized as contemporary society and culture become increasingly complicated and/or assimilated [8].

Traditionally, Korea and Japan share similar cultural traits. Both belong to collectivistic and high-context cultures, and both observe Confucianism ([5], [17], [18]). The similarities in their cultural traits may be due in part to their regional and historical closeness. However, there are clear differences between Korea and Japan. Some scholars have argued that Japanese culture exhibits high level of individualistic as well as 
collectivistic cultural traits because of the country's extraordinary economic development in recent decades [3]. Korean culture has kept the strictest collectivistic cultures among the three Confucianism-based Asian countries, China, Korea, and Japan ([17]).

The study explores Twitter use in Korea and Japan separately to gain a better understanding of the effects of Twitter on each individual society. Overall, Korea and Japan have both cultural similarities and differences based on individualism-collectivism dimension. Additionally, the currently well-developed IT environment would contribute to forming similarities and differences in the use of IT services. To clarify these similarities and differences, we examined published information from Twitter profiles, including Tweets, followers/followings, retweets, and profile information. In particular, we noted that geographic information is one of the most important pieces of personal information for identifying a person in interpersonal communication. For data collection, we used software which we developed for this analysis. The study also analyzes Tweets in terms of nine categories and compares them by nationality. As mentioned above, this study contributes to the extension of empirical research of social media by providing a cross-national comparison of Twitter use between Korea and Japan.

\section{RELATED WORK}

Despite collectivistic cultures, Korea and Japan have different cultural traits [3]-[4]. Korean culture reflects simple collectivism, which means that depending on the situation, ingroups are adjusted flexibly and that members have multiple ingroups. By contrast, Japanese culture reflects contextual collectivism, which means that members from specific ingroups beyond given context and situations [4]. This study attempts to find illumination using the framework, simple and contextual collectivism and in doing so, it will contribute to extension of literature relating to simple-and contextualcollectivism.

In terms of cross-cultural research, there are few previous studies related to new media use between Korea and Japan despite their economic and geographical closeness. Meanwhile, Lee et al. (2002) explored the comparison of mobile Internet between the two countries and left several interesting implications [9]. For instance, the study showed that Korean users tend to mainly use mobile Internet for entertainment, such as download service or online game, whereas Japanese users tend to mostly use it for email service. Lee et al. claimed that emailing symbolizes information-oriented usage, yet we paid attention to the communication pattern of email with targeted partners more than its purpose. That is, their results imply that Japanese users have not willingly extended their communication partners using new media. Koreans, in contrast, show a tolerance for in-grouping acquaintances or even the unfamiliar: for instance, enjoying online games with networked players, including the known and the unknown.
Based on the theoretical frame and previous research, this study assumes that Koreans are more willing to extend connections and have more interested in interactive communications through Twitter, compared with Japanese who are stricter to increase in-group and socializing boundaries.

\section{METHOD}

\subsection{Data Collection}

We collected unique IDs from Twitter's public timeline for approximately seven hours from August 18-19, 2010. We filtered the data by using an API-based software. This process left us with 300 Korean and 300 Japanese Tweets by unique IDs (for detailed procedures, see [12]. In addition, for three days, we collected profile information and Tweets for these 600 unique IDs by using the Twitter API (Application Program Interface) and a Twitter scraper that we developed. We excluded some IDs because their tweets were commercials. Consequently, we analyzed 286 Korean and 283 Japanese IDs (Table 1). When counting the number of Tweets, followers, and followings, we were unable to determine when each ID was created. Although we could not ascertain the history of each Twitter ID, we assumed that this limitation would have little influence on the results. It is because the remarkable increase in the number of Twitter accounts occurred around a short time both in Korea and in Japan ${ }^{1}$ and the fact allowed us to assume that most of Twitter IDs created in a similar period on average. Additionally, there were no major variables related to the date of ID creation.

Table 1. Participants and their Twitter use

\begin{tabular}{|c|c|c|c|c|c|c|}
\hline & \multicolumn{6}{|c|}{ Twitter Use } \\
\hline & \multicolumn{3}{|c|}{ Korea $(N=286)$} & \multicolumn{3}{|c|}{ Japan $(N=283)$} \\
\hline & $\begin{array}{l}\text { Valid } \\
\text { no. }\end{array}$ & Male & Female & $\begin{array}{l}\text { Valid } \\
\text { no. }\end{array}$ & Male & Female \\
\hline Gender & 165 & $\begin{array}{l}106 \\
(64 \%)\end{array}$ & $\begin{array}{l}59 \\
(36 \%)\end{array}$ & 204 & $\begin{array}{l}145 \\
(71 \%)\end{array}$ & $\begin{array}{l}59 \\
(29 \%)\end{array}$ \\
\hline Reciprocity & & $76.40 \%$ & & & $73.80 \%$ & \\
\hline $\begin{array}{l}\text { No. of } \\
\text { Tweets }\end{array}$ & & 4292 & & & 9347 & \\
\hline $\begin{array}{l}\text { No. of } \\
\text { followers }\end{array}$ & & 1047 & & & 323 & \\
\hline $\begin{array}{l}\text { No. of } \\
\text { followings }\end{array}$ & & 980 & & & 285 & \\
\hline $\begin{array}{l}\text { Pieces of } \\
\text { geographic } \\
\text { information }\end{array}$ & & $166(58 \%$ & & & $143(51 \%$ & \\
\hline $\begin{array}{l}\text { No. of } \\
\text { metropolitans }\end{array}$ & 154 & $111(72 \%$ & & 143 & $68(48 \%)$ & \\
\hline
\end{tabular}

* The percentages for "gender" and "no. of metropolitans" were calculated using only valid cases.

\footnotetext{
${ }^{1}$ For publicly available statistical information, see http://whatjapanthinks.com/2010/06/29/twitter-japan-statisticsone-in-three-tweeting-daily-two-thirds-joined-this-year/ (Japanese information) and http://blog.oiko.cc/post/1139287720/153 (Korean information).
} 
We conducted a content analysis of Tweets by the unique IDs. We selected every $10^{\text {th }}$ tweet until we collected 10 Tweets for each ID. If we were unable to collect 10 Tweets from an ID's ego data because of a lack of original Tweets, we excluded that ID from the sample. We based this on the assumption that a user who posted fewer than 100 tweets at that point in time is an inactive Twitter user. We collected 2,450 Korean Tweets and 2,730 Japanese Tweets for the final content analysis.

\subsection{Measurement and the Coding Process}

To compare Korean users with their Japanese counterparts, we conducted independent samples $t$-tests for tweets, retweets, the rate of reciprocity, and followings/followers. We also compared the results of the content analysis following [11]. For each message, Naaman et al. created nine categories as follows: a) information sharing (IS), i.e., providing information; b) selfpromotion (SP), i.e., promoting works of the author; c) opinions/complaints (OC), i.e., providing opinions or complaints; d) statement and random thoughts (RT), i.e., talking to oneself; e) me now (ME), i.e., describing what the author is doing or feeling; f) question to followers (QF), i.e., inducing others to reply; g) presence maintenance (PM), i.e., describing the current situation or status around the author; $h$ ) anecdote (me) (AM), i.e., conveying author's past episodes; and, i) anecdote (others) (AO), i.e., conveying others' past episodes.

Tweets were coded into the categories by four coders. Two coders analyzed Korean Tweets, and the remaining two, Japanese Tweets. These coders were trained together, and afterward, they coded separately. Following [9], we allowed the coders to assign multiple categories to each message, and thus, the total percentage for each sample was greater than $100 \%$.

\section{RESULTS}

\subsection{Differences in Twitter Use between Korea and Japan}

First, we examined the proportion of reciprocal connections, which was $76.4 \%$ for Korean sample and $73.8 \%$ for Japanese sample. Although Korean IDs showed a slightly higher proportion of reciprocal connections, the results of the independent samples $t$-test do not indicate a significant difference.

Second, we compared the number of Tweets through the independent samples $t$-test. There was a significant difference in the number of Tweets between Korean and Japanese samples $(t(468)=-5.858, p<.001)$. During the data collection period, Koreans posted approximately 4,292 Tweets, and the Japanese, approximately 9,347. Thus, Japanese Tweets outnumbered Korean Tweets by 2 to 1 .

Third, we conducted independent samples $t$-tests to examine the numbers of followers and followings. There were significant differences in both the number of followers $(t(321)=$ $4.963, p<.001)$ and the number of followings $(t(317)=4.993$, $p<.001)$ between the two samples. Korean users had approximately 1,047 followers and 980 followings, whereas Japanese users had approximately 323 followers and 285 followings. Thus, Korean sample had approximately three times as many followers and followings as Japanese ones. Japanese users posted significantly more Tweets, whereas Korean users had significantly more connections. This is noteworthy in that both measurements represent positive Twitter use for activity and interactivity.

\subsection{Effects of the Disclosure of Geographic Information on Twitter Use}

We compared the Twitter users who disclosed their geographic location on their profile with those who did not by using the independent samples $t$-test. As shown in Table 2, Korean users who disclosed their geographic location had significantly more followers $(t(261)=3.336, p<.005, M=1408$, $S D=2834.2)$ and followings $(t(251)=3.382, p<.005, M=1328$, $S D=2744.4)$ than those who did not $(M=548, S D=2472.2$; $M=500, S D=1315.0$, respectively). The result indicates that users who disclose their geographic information have a more positive attitude to make connections on Twitter than those who do not. There were no significant differences in the total number of Tweets and the proportion of reciprocal connections.

Table 2. Twitter use by geographic information

\begin{tabular}{lllll}
\hline \multicolumn{5}{l}{ Twitter Use } \\
& \multicolumn{2}{l}{ Korea $(N=286)$} & \multicolumn{2}{l}{ Japan $(N=283)$} \\
\cline { 2 - 5 } & Info & $\begin{array}{l}\text { No } \\
\text { info }\end{array}$ & Info & No info \\
\cline { 2 - 5 } Geographic & 166 & 120 & 143 & 140 \\
information & $(58 \%)$ & $(42 \%)$ & $(51 \%)$ & $(49 \%)$ \\
$\begin{array}{l}\text { Reciprocity } \\
\text { No. of } \\
\text { Tweets }\end{array}$ & $504 \%$ & $72 \%$ & $72 \%$ & $75 \%$ \\
$\begin{array}{l}\text { No. of } \\
\text { followers }\end{array}$ & 1408 & 548 & 8134 & 10585 \\
$\begin{array}{l}\text { No. of } \\
\text { followings }\end{array}$ & 1327 & 500 & 308 & 293 \\
\hline
\end{tabular}

In Japanese sample, for all the variables, there were no significant differences between the users who disclosed their geographic location and those who did not. It may imply that Japanese users are less likely to be interested in connections on Twitter in general. Given that geographic information is related to self-disclosure that initiates interactions and relationships [1], the result corresponds to Japanese negative attitude to extending relationships.

To sum up, we explored the role of geographic information on the Twitter profile in Twitter use. The results indicate that for Korea, geographic information was positively related to the numbers of followers and followings. Geographic information had no significant effect on Twitter use in Japan and no significant effect on the number of Tweets for both Korea and Japan. Self-disclosure may be positively related to the development of social relationships. The results for Korean users provide support for the effect of self-disclosure on social 
relationships on Twitter. That is, geographic information provides a communication cue for visitors to the Twitter profile, and consequently, Twitter users who show a high level of selfdisclosure are more likely to have Twitter connections.

On the other hand, geographic information had no significant effect on Japanese users' Twitter connections. This may be because Twitter users in Japan are more likely to restrict their Twitter connections within an area than those in Korea. That is, connections in a narrow area would remove the difference of area distribution among Japanese users and accordingly, it could not be a significant variable to affect Twitter use. It may also be because self-disclosure-which is used mainly for initiating new social relationships between zero-history interactants - is not necessary in a society in which the ingroup boundary is predetermined and new social relationships are rare.

\subsection{The Analysis of Tweet Messages}

We categorized the Tweets of Korean and Japanese users in terms of nine themes. In the sample, Korean users were more likely to be engaged in information sharing (IS, 25.5\%), me now (ME, 23.9\%), and questions for followers (QF, 4.9\%) than Japanese users $(12.4 \%, 17.5 \%$, and $2.6 \%$, respectively). Korean users were slightly more likely to post anecdotes-others (AO, $1.5 \%)$ than Japanese users $(0.8 \%)$. In contrast, Japanese users were more likely to post statements/random thoughts (RT, $30.8 \%$ ), opinions/complaints (OC, 20.8\%), and anecdotes-me (AM, 11\%) than Korean users $(16.4 \%, 17.4 \%$, and $6.8 \%$, respectively). Further, Japanese users were slightly more likely to post Tweets reflecting self-promotion (SP, 1.4\%) and presence maintenance (PM, 2.8\%) than Korean users $(0.9 \%$ and $2.7 \%$, respectively). Figure 1 shows the types of Tweets.

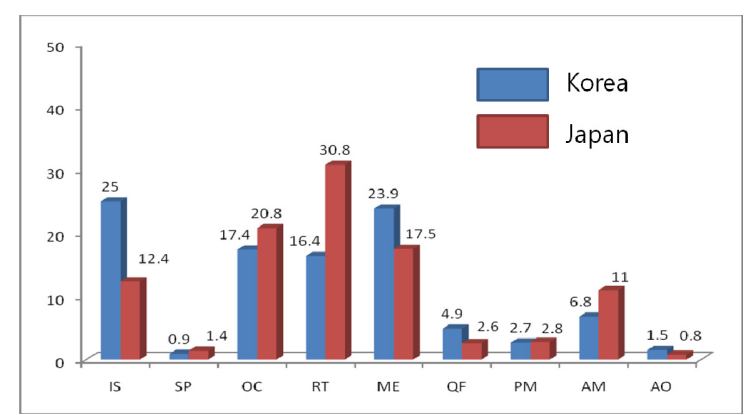

Fig. 1. Types of tweets: Korea vs. Japan

IS: Information Sharing; SP: Self-Promotion; OC: Opinions/Complaints; RT: Statements/Random Thoughts; ME: Me Now; QF: Questions for Followers; PM: Presence Maintenance; AM: Anecdotes-Me; AO: Anecdotes-Others.

The results of the content analysis indicate that for Korean users, the primary purpose of using Twitter was information sharing, a goal-oriented communication action, and that for Japanese users, it was personal graffiti disappearing (or unnecessary) communication context. This difference can be explained by the differences in collectivistic cultural traits between the two countries: simple vs. contextual collectivism. Koreans tend to form or modify the in-group boundary in a given situation, whereas the Japanese tend to adhere to a predefined boundary regardless of the situation (Gudykunst et al., 1987). For Koreans, Twitter friends-followings/followerscan be included as in-group members within the Twitter boundary. Considering their Twitter friends as in-group members, Koreans are likely to value sincere interactions. At the same time, they may perceive that Twitter connections do not ensure relational faith, which is consistent with offline friendships. Information sharing enables users to provide Twitter friends with benefits by delivering useful information. It can also allow them to involve a broad range of individuals in their Twitter activity regardless of the level of their intimacy with the author and without disclosing sensitive personal information. Thus, information sharing is an effective communication strategy both for facilitating faithful interactions and for maintaining individuality.

For Twitter users in Japan, Twitter connections may simply be ephemeral online connections that are disconnected from offline relationships, and thus, Twitter connections are not part of the in-group. The lack of intimate messages is consistent with Japanese users' attempt to prevent out-group members from accessing the Twitter timeline. Most of the Tweets by Japanese users were statements and random thoughts, which suggests that Twitter users in Japan are not likely to engage readers through Tweets. Their messages seemed as if they were talking to themselves in public. That is, one would have difficulty understanding their messages if one did not have access to personal information on the author. Thus, Twitter users in Japan are likely to engage in self-disclosure while excluding out-group members from their personal lives. Thus, the results indicate that Twitter users build communication strategies based on the type of message they wish to disclose and that such strategies tend to reflect their cultural orientation.

\section{CONCLUSION}

This study examined and compared the use of social media between the Koreans and Japanese. Further, this study attempts to comprehend the features of socializing on social media between the two countries. The results of this study demonstrate that users build their own communication strategies by reflecting their cultural orientation as well as adopting global media attributes.

Koreans' tolerance for flexible in-group boundaries in simple collectivism is parallel with the large amount of Twitter connections. Additionally, the larger amount of Twitter messages among Japanese remind us of the early adoption of mobile Internet as well as the recent motive of Twitter growth, such as crisis communication during 3.11 earthquakes [15]. Contrasted with Japanese users, Koreans are more likely to use geographic information for the increase of connections and to post messages intriguing interactions. 
These results are paralleled with cultural traits of each country, simple and contextual collectivism. Accordingly, this study claims that traditional culture is more or less engaged in the adaptation of the new media. Therefore, top management staff in Twitter.com needs to recognize that a national/cultural attitude toward technology might be significant factor for the success of social networking services in future.

Although this study contributes to extending literature of cross-national/cultural research in new media use, it has limited sampling. We collected data during a certain period, and this does not represent all users in the two countries. Furthermore, the rapid shift of Twitter use may confine the results to a certain period. The comparison between the two samples was also simplistic. It can entail a doubt on the reliability of the results. However, the consistent findings provide value to this study as a heuristic research that reveals there is no decrease in the effect of local culture on communication behaviors.

\section{ACKNOWLEDGEMENTS}

The authors are grateful for valuable feedback from researchers at the World Class University Webometrics Institute and acknowledge partial support from the WCU program (Grant No. 515-82-06574). The authors wish to thank Steven Sams, Jiyoung Park, Jiyoung Kim, and Tetsuro Kobayashi for their assistance during data collection and comments on the earlier versions of this paper.

\section{REFERENCES}

[1] N. Collins and L. Miller, "Self-Disclosure and Liking: A Meta-Analytic Review," Psychological Bulletin, vol. 116, no. 3, 1994, pp. 457-475.

[2] C. Geertz, The Interpretation of Cultures: Selected Essays, The 2000 Edition. Basic Books, New York, 1973.

[3] W. Gudykunst and T. Nishida, "The Influence of Cultural Variability on Perceptions of Communication Behavior Associated with Relationship Terms," Human Communication Research, vol. 13, no. 2, 1986, pp. 147166.

[4] W. Gudykunst, Y. Yoon, and T. Nishida. "The Influence of Individualism-Collectivism on Perceptions of Communication in Ingroup and Outgroup Relationships," Communication Monographs, vol. 54, 1987, pp. 295-305.

[5] G. H. Hofstede, Culture's Consequences: Comparing Values, Behaviors, Institutions, and Organizations across Nations. 2nd ed. Sage Publications, Thousand Oaks, CA, 2001.

[6] B. Jansen, K. Sobel, and G. Cook, "Classifying Ecommerce Information Sharing Behavior by Youths on Social Networking Sites," Journal of Information Science, vol. 37, no. 2, 2011, pp. 120-136.

[7] M. Kang, B. Kim, P. Gloorr, and G-W, Bock, "Understanding the Effect of Social Networks on User Behaviors in Community Driven Knowledge Services,"
Journal of the American Society for Information Science and Technology, vol. 62, no. 6, 2011, pp. 1066-1074.

[8] B. Kirkman, K. Lowe, and C, Gibson, "A Quarter Century of Culture's Consequences: A Review of Empirical Research Incorporating Hofstede's Cultural Values Framework," Journal of International Business Studies, vol. 36, no. 3, 2006, pp. 285-320.

[9] Y. Lee, J. Kim, I, Lee, and H. Kim, “A Cross-Cultural Study on the Value Structure of Mobile Internet Usage: Comparison between Korea and Japan," Journal of Electronic Commerce Research, vol. 3, no. 4, 2002, pp. 227-239.

[10] U. Matzat, "Disciplinary Differences in the Use of Internet Discussion Groups: Differential Communication Needs or Trust Problems?," Journal of Information Science, vol. 35, 2009, pp. 613-631.

[11] Y. M. Mei, S. T., Lee, and S. Al-Hawamdeh, "Formulating a Communication Strategy for Effective Knowledge Sharing." Journal of Information Science, vol. 30, no. 1, 2004, pp. 12-22.

[12] M. Naaman, J. Boase, and C-H. Lai, "Is it really about me? Message content in social awareness streams." Proc. CSCW'10, 2010.

[13] S. Sams, Y. S. Lim, and H. W. Park, "E-Research Applications for Tracking Online Socio-Political Capital in the Asia-Pacific Region," Asian Journal of Communication, vol. 21, no. 5, 2011, pp. 450-466.

[14] U. Schimmack, S. Oishi, and D. Diener, "Individualism: A Valid and Important Dimension of Cultural Differences between Nations," Personality and Social Psychology Review, vol. 9, no. 1, 2005, pp. 17-31.

[15] C. Taylor, "Twitter Users React to Massive Quake, Tsunami in Japan," Mashable. (2011). Retrieved 17 December, 2011, from http://mashable.com/2011/03/11/ japan-tsunami/.

[16] M. Thelwall and D. Wilkinson, "Public Dialogs in Social Network Sites: What is their Purpose?" Journal of the American Society for Information Science and Technology, vol. 61, no. 2, 2010, pp. 392-404

[17] S. Ting-Toomey, "Intercultural Conflict Styles: A FaceNegotiation Theory." In Theories in Intercultural Communication, eds, 1988.

[18] H. Triandis, Collectivism and Individualism, Westview Press, Boulder, Colorado, 1955.

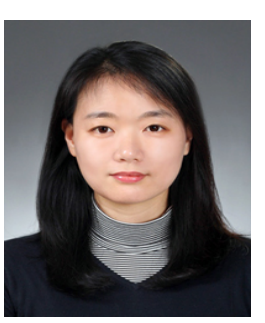

Seong Eun Cho

She is a research fellow at Korea Information Society Development Institute. Her research areas include social media, online socializing, crosscultural and intercultural communication, and new media and digital technology. She acknowledges that this research has been carried out during her stay in CyberEmotions Research Institute at YeungNam University. 


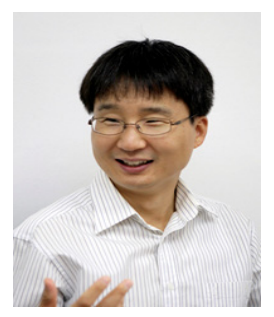

\section{Han-Woo Park}

$\mathrm{He}$ is currently working as an associate professor in the Department of Media \& Communication, YeungNam University, South Korea. Over the past several years, he has contributed important works in Webometrics from the perspective of social-network analysis. He was formerly principal investigator for an international research project entitled 'Investigating internet-based politics using e-research tools' (April 2008 to August 2011), funded by the Korean government's WCU (World Class University) Program. He is a Director of Cyber Emotions Research Institute and a President of Asia Triple Helix Society. 